\title{
ERRATUM TO: SOLUTIONS TO FOUR OPEN PROBLEMS ON QUORUM COLORINGS OF GRAPHS
}

\author{
RAFIK SAHBI*[B]
}

\begin{abstract}
The purpose of this erratum is to correct the references to some theorems and propositions in the original article. Also, for the sake of clarity, the author would like to modify certain passages of the second paragraph of Theorem 2.6 proof's by adding a few short sentences and deleting others in order to remove the redundancy.
\end{abstract}

Received September 8, 2021. Accepted September 8, 2021.

Erratum to: RAIRO-Oper. Res. 55 (2021) 2385-2394. https://doi.org/10.1051/ro/2021116

\section{LIST OF CORRECTIONS AND MODIFICATIONS}

(1) Page 2387, line 25: Change "Theorem 2" to "Theorem 2.2".

(2) Page 2387, line 26: Change "Conjecture 2" to "Conjecture 2.1".

(3) Page 2388, line 17: Change "Moreover" to "Furthermore".

(4) Page 2388, line 18: Change "Proposition 2" to "Proposition 2.4".

(5) Page 2388, between lines 18 and 19: Delete the sentence "Furthermore, since $\delta(G) \geq 2$ and since according to Proposition $2 G\left[V_{i}\right]$ is connected, we get $\left|V_{i}\right| \geq d_{V_{i}}(v)+1 \geq 2$, for all $i \in\{1,2\}$ and $v \in V_{i}$." because its conclusion " $\left|V_{i}\right| \geq 2$ " is deduced just before.

(6) Page 2388, line 20: Add the sentence "For an arbitrary chosen vertex $v \in V$, set" between "." and " $V_{i}^{\prime}=$ $V_{i} \backslash\{v\}$ and $V_{3-i}^{\prime}=V_{3-i} \cup\{v\}$." because the vertex $v$ has not been defined.

(7) Page 2388, line 20: Change "any" to "the" because the vertex $v$ is now chosen in the item 6.

(8) Page 2388, line 23: Add the sentence "according to Proposition 2.5" between "connected" and the first comma "," because Proposition 2.5 justifies the connectivity of $G\left[V_{i}\right]$.

(9) Page 2388, between the lines 11 and 23: Reverse the order of the two paragraphs of the proof to match the new first and second paragraphs respectively to the necessary and sufficient conditions as stated in Theorem 2.6.

By applying the above nine items, the proof of Theorem 2.6 becomes as follows.

Proof. Assume that $\psi_{q}(G) \geq 2$ and let $\pi=\left\{V_{1}, V_{2}\right\}$ be a quorum coloring of $G$ of order 2 . Then $d_{V_{i}}(v)+1 \geq$ $d_{V_{3-i}}(v)$, for every $i \in\{1,2\}$ and $v \in V_{i}$. Furthermore, since $G$ does not have leaves, then we have by Proposition 2.4 that $\left|V_{i}\right| \geq 2$ for every $i \in\{1,2\}$. Let us show that $e\left(V_{1}, V_{2}\right)$ is a critical (-1)-cutset. For an arbitrarily chosen vertex $v \in V_{i}$, set $V_{i}^{\prime}=V_{i} \backslash\{v\}$ and $V_{3-i}^{\prime}=V_{3-i} \cup\{v\}$. Therefore, by moving the vertex $v$ from $V_{i}$ to $V_{3-i}$, we obtain that $\left|e\left(V_{i}^{\prime}, V_{3-i}^{\prime}\right)\right|=\left|e\left(V_{i}, V_{3-i}\right)\right|-d_{V_{3-i}}(v)+d_{V_{i}}(v)$. By using the inequality $d_{V_{i}}(v)+1 \geq d_{V_{3-i}}(v)$, it

Department of the Preparatory Training Algiers Higher School of Applied Sciences, B.P. 474, Martyrs Square, Algiers 16001, Algeria.

*Corresponding author: r.sahbi@g.essa-alger.edu.dz

(C) The authors. Published by EDP Sciences, ROADEF, SMAI 2021

This is an Open Access article distributed under the terms of the Creative Commons Attribution License (https://creativecommons.org/licenses/by/4.0), which permits unrestricted use, distribution, and reproduction in any medium, provided the original work is properly cited. 
follows that $\left|e\left(V_{i}^{\prime}, V_{3-i}^{\prime}\right)\right| \geq\left|e\left(V_{i}, V_{3-i}\right)\right|-1$, which means that $\left|e\left(V_{1}, V_{2}\right)\right|$ decreased by at most 1 . Moreover, since $G\left[V_{i}\right]$ is connected according to Proposition 2.5, then $e\left(V_{1}, V_{2}\right)$ is a minimal (-1)-cutset. As a result, $e\left(V_{1}, V_{2}\right)$ is a critical $(-1)$-cutset.

Conversely, let $e\left(V_{1}, V_{2}\right)$ be a critical (-1)-cutset of $G$. Then, for every $i \in\{1,2\}$, moving any vertex from $V_{i}$ to $V_{3-i}$ decreases the size of $e\left(V_{1}, V_{2}\right)$ by at most one edge. Thus, by putting $V_{i}^{\prime}=V_{i} \backslash\{v\}$ and $V_{3-i}^{\prime}=V_{3-i} \cup\{v\}$ for some $i \in\{1,2\}$ and some vertex $v \in V_{i}$, we have $\left|V_{i}^{\prime}\right| \geq 1$ (that is, $V_{i}^{\prime} \neq \emptyset$ ) and $\left|e\left(V_{i}^{\prime}, V_{3-i}^{\prime}\right)\right|=\left|e\left(V_{i}, V_{3-i}\right)\right|-$ $d_{V_{3-i}}(v)+d_{V_{i}}(v) \geq\left|e\left(V_{i}, V_{3-i}\right)\right|-1$. By eliminating $\left|e\left(V_{i}, V_{3-i}\right)\right|$ on both sides of the inequality, we obtain that $d_{V_{i}}(v)+1 \geq d_{V_{3-i}}(v)$, which means that $\left\{V_{1}, V_{2}\right\}$ is a quorum coloring of $G$ of order 2. Consequently, $\psi_{q}(G) \geq 2$.

(10) Page 2388, line 38: Change "Proposition 2" to "Proposition 2.10".

(11) Page 2389, line 1: Change "Proposition 2" to "Proposition 2.10".

(12) Page 2389, line 14: Change "Proposition 2" to "Proposition 2.10".

(13) Page 2389, line 29: Change "Proposition 2" to "Proposition 2.10".

(14) Page 2390, line 3: Change "Proposition 1" to "Proposition 1.3".

(15) Page 2390, line 4: Change "Proposition 1" to "Proposition 1.4".

(16) Page 2390, line 12: Change "Theorem 1" to "Theorem 1.1".

(17) Page 2390, line 25: Change "Proposition 1" to "Proposition 1.3".

(18) Page 2391, line 21: Change "Proposition 4" to "Proposition 4.1".

(19) Page 2391, line 23: Change "Proposition 4" to "Proposition 4.1".

(20) Page 2391, line 30: Change "Proposition 4" to "Proposition 4.1".

(21) Page 2391, line 35: Change "Proposition 1" to "Proposition 1.3".

(22) Page 2392, line 7: Change "Proposition 5" to "Proposition 5.1".

(23) Page 2392, line 19: Change "Proposition 5" to "Proposition 5.1". 